\title{
Gluconeogenesis from Lactate in the Small Intestinal Mucosa of Suckling Rats
}

\author{
PETER HAHN AND HO WEI-NING \\ Departments of Obstetrics and Gynecology and Pediatrics, University of British Columbia, The Research Centre, \\ Vancouver, B. C. V5Z 4H4, Canada
}

\begin{abstract}
Glucose formation from uniformly labeled ${ }^{14} \mathrm{C}$-lactate was studied in the small intestinal mucosa of rats and rabbits. It was found to occur in infant but not in adult (weaned) animals and to be increased by the presence of dibutyryl cyclic AMP or tetradecyl glycidic acid. Similarly the formation of glyceride glycerol was enhanced by tetradecyl glycidic acid but not by glucagon or cyclic AMP. The glycogen content of the intestinal mucosa was always low, but increased significantly at the time of weaning. It is suggested that gluconeogenesis occurs in the small intestinal mucosa of infant rodents to supply glucose to the muscular part of the small intestine. (Pediatr Res 20: 13211323, 1986)
\end{abstract}

\section{Abbreviations}

PEPcK, phosphoenolpyruvate carboxykinase

FDPase, fructose diphosphatase

TDGA, tetradecylglycidic acid

KRB, Krebs-Ringer bicarbonate

We showed in previous work that the mucosa of the small intestine of suckling rats contains both phosphoenol-pyruvate carboxykinase and fructose biphosphatase activities and that at the time of weaning, both enzyme activities fall to very low levels $(1,2)$. A similar developmental pattern has been described for glucose-6-phosphatase (3). The present studies were conducted to determine whether the presence of these three enzymes in the mucosa of suckling rats is related to the formation of glucose.

\section{MATERIALS AND METHODS}

Wistar rats bred in our laboratory were used throughout. Litters were reduced to nine pups 1 day after delivery and were kept with the mother until the time of sacrifice. After decapitation the gut was rapidly removed and immediately rinsed with ice-cold saline. The small intestine was placed on a paper towel and the mucosa was expressed from it using a metal spatula. The mucosae from the whole litter, or preferably from two litters, were placed on ice-cooled aluminium dishes, mixed, and distributed in lots of about $300 \mathrm{mg}$ into Ehrlenmeyer flasks containing $5 \mathrm{ml} \mathrm{Krebs-Ringer} \mathrm{bicarbonate} \mathrm{buffer} \mathrm{and} \mathrm{substrates} \mathrm{and} \mathrm{other}$ substances as described below. Throughout the incubation, vessels were aerated with $95 \%$ oxygen and $5 \% \mathrm{CO}_{2}$.

The glycogen content of the mucosa was determined by the anthrone method (4). PEPcK activity was determined in the high speed supernatant of a mucosal homogenate in sucrose $(0.25 \mathrm{M})$,

Received April 11, 1986; accepted July 31, 1986.

Correspondence Dr. Peter Hahn, The Research Centre, 178-950 W. 28th Avenue, Vancouver, B. C. V5Z 4H4, Canada.

Supported by a grant from Medical Research Council (Canada).
EGTA $(1.0 \mathrm{mM})$ and Tris $(3.4 \mathrm{mM}), \mathrm{pH} 7.4$ at the start and the end of the incubation period, using previously described methods (1). FDPase activity was determined in the same supernatant as described (2). Tissues were incubated for up to $2 \mathrm{~h}$. Samples of the incubation medium were taken every hour. The KRB incubation medium contained $10 \mathrm{mM}$ glutamate and $10 \mathrm{mM}$ lactate with a specific activity of $100 \mathrm{DPM} / 6.8 \mathrm{nmol}$. The following additions were made: glucagon $\left(10^{-7} \mathrm{M}\right)$, dibutyryl cyclic AMP $(1 \mu \mathrm{M})$, and TDGA $(0.1 \mathrm{mg} / 5 \mathrm{ml})$ an inhibitor of carnitinepalmitoyl transferase (5). Other additions are shown in the appropriate figures.

Some experiments were performed using rabbits. In contrast to rats, PEPcK is present not only in the cytoplasm but also in the mitochondria (6). Hence in this species these particles were isolated by spinning the homogenate at $2000 \times g$ and the supernatant at $8000 \times g$ for $20 \mathrm{~min}$, the pellet being considered to consist of mitochondria. Radioactive glucose was isolated on Amberlite MB3 and counted in a Beckman scintillation counter. Results were expressed in $\mathrm{nmol} / \mathrm{mg}$ wet weight. Statistical significance was evaluated using the paired $t$ test.

\section{RESULTS}

The glycogen content of the mucosa is uniformly low during postnatal development of the rat and increases after weaning (Table 1). It falls to about half the initial value during incubation (Table 2). The activity of PEPcK decreases somewhat during the incubation period but even at the end of $2 \mathrm{~h}$ of incubation, activity was high compared to the adult mucosa. Similarly FDPase activity decreased but did not disappear (not shown).

Glucose formation from labelled lactate occurred only in suckling rats and not in weaned animals (Fig. 1). It was increased

Table 1. Glycogen content in the proximal half of the small intestinal mucosa of infant and adult rats*

\begin{tabular}{ccc}
\hline & $\begin{array}{c}\text { Glycogen } \\
\text { Age (days) }\end{array}$ & $n$ \\
\hline $1-5$ & $515^{a}$ & 5 \\
$7-15$ & $357^{a}$ & 6 \\
$35-60$ & $894^{b}$ & 10 \\
\hline
\end{tabular}

* Data with different superscripts differ from each other for $p<0.01$.

Table 2. Changes in glycogen content and phosphoenolpyruvate carboxykinase activity in the proximal intestinal mucosa of 12-day-old rats during incubation

\begin{tabular}{lcccc} 
& $\begin{array}{c}\text { Glycogen } \\
(\mathrm{mg} / 100 \mathrm{~g} \text { wet } \mathrm{wt})\end{array}$ & $n$ & $\begin{array}{c}\text { PEPcK } \\
(\mathrm{nmol} / \mathrm{min} / \mathrm{mg} \text { protein })\end{array}$ & $n$ \\
\hline Start & $36 \pm 2.6$ & 4 & $75 \pm 8$ & 4 \\
1 h incubation & $30 \pm 2.0$ & 4 & $74 \pm 8$ & 4 \\
2 h incubation & $16.5 \pm 1.5$ & 4 & $50 \pm 4$ & 4 \\
\hline
\end{tabular}


by the addition of cyclic AMP and, surprisingly, also by TDGA (Fig. 2). Triglyceride glycerol formation from labeled lactate also occurred essentially only in the mucosa from suckling rats and was not found in weaned rats. Only PEPcK is required for this process, which probably explains why this rate was greater than that of glucose formation (Fig. 3). Cyclic AMP (not shown) and glucagon had no effect on glycerol synthesis but TDGA increased it. In rabbit liver, in contrast to the rat organ, PEPcK is found in both mitochondria and cytoplasm (6). Hence, the intestinal mucosa of rabbits was examined for both a mitochondrial and a cytoplasmic enzyme. PEPcK was found in both parts of the cells. However, cytoplasmic activity is high in the cytoplasm of suckling rabbits and minimal in weaned animals. The mitochondrial enzyme shows highest activity soon after birth and also falls during further development (Fig. 4).

Glucose formation in the mucosa obtained from suckling rabbits also was significantly higher than in that from weaned animals (Fig. 5). The effect of weaning to a high fat or high carbohydrate diet on day 18 on intestinal PEPcK activity is shown in Figure 6. It is apparent that early weaning to a high fat diet delays the decrease in enzyme activity but does not prevent it. This is similar to the finding with fructose-biphosphatase (2) reported earlier.

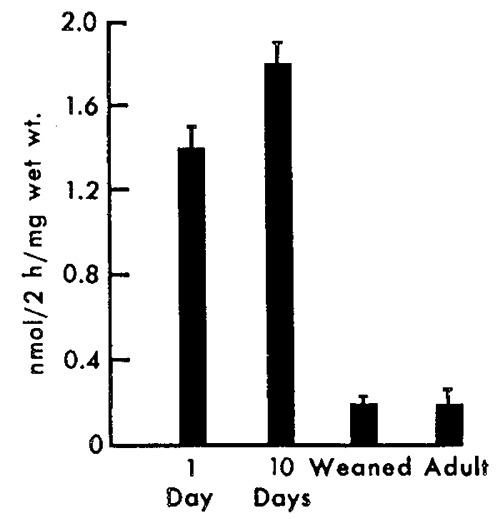

Fig. 1. Glucose formation by the proximal half of the small intestinal mucosa obtained from rats aged 1,10 , and 21 (weaned) days and adult animals. Means and SE from 16 experiments consisting of 16 individual rats per experiment. Incubation: $2 \mathrm{~h}$. The difference between days 1 or 10 and older animals is significant for $p<0.001$.

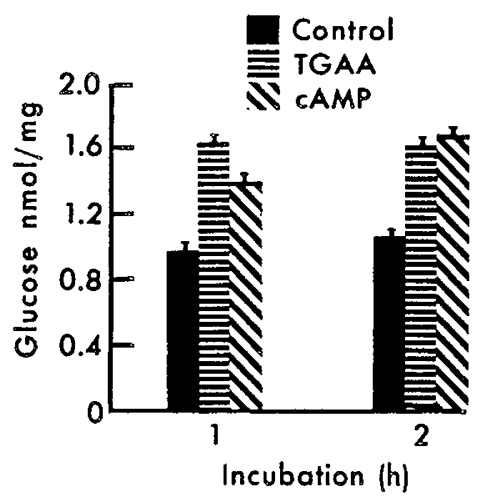

Fig. 2. The effect of TDGA (TGAA) and dibutyryl cyclic AMP (OAMP) on glucose formation from lactate by the small intestinal mucosa of 12-day-old rats after 1 and $2 \mathrm{~h}$ of incubation. The effects of both TDGA and O-AMP are significant for $p<0.002$. Means and SE from 16 experiments. Abscissa: hours of incubation; black: control; shaded horizontal: TDGA; shaded slanted: cyclic AMP.

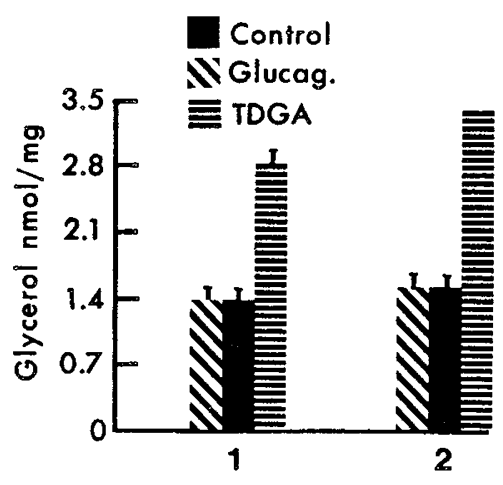

Fig. 3. Triglyceride glycerol formation from labeled lactate by the small intestinal mucosa of 12-day-old rats. Means and SE from 10 experiments. Black: control; shaded slanted: glucagon; shaded horizontal: TDGA. The effect of TDGA is significant for $p<0.001$.

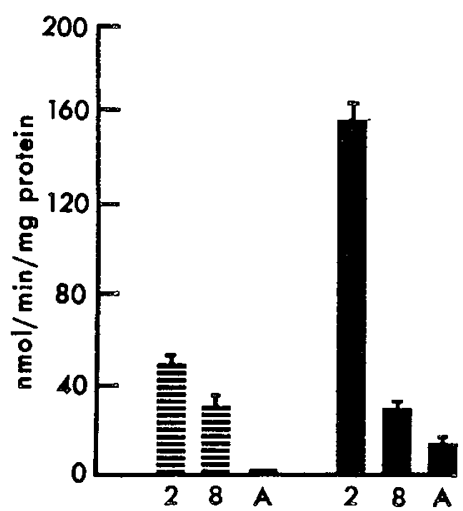

Fig. 4. The development of phosphoenolpyruvate carboxykinase activity in the cytoplasm and mitochondria of the proximal half of the small intestinal mucosa of rabbits. Incubation: $1 \mathrm{~h}$. Means and SE of six experiments in each age group. Abscissa: age in days; $A$ : adult; shaded three columns (left): cytoplasm; black three columns (right): mitochondria.

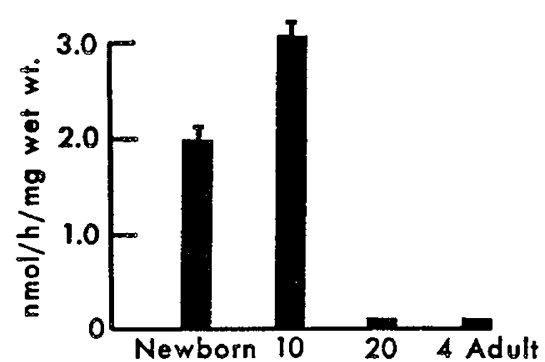

Fig. 5. Glucose formation from labeled lactate by the small intestinal mucosa of rabbits. Means and SE of eight experiments. $p<0.001$ between infant and weaned animals. Incubation: $1 \mathrm{~h}$.

\section{DISCUSSION}

There is only one report in the literature regarding the glycogen content of the developing gut (7). It describes the accumulation of glucose on the mucosal side of the everted sac from neonatal but not adult rats and attempts to correlate this with the amount of glycogen in the intestinal wall (not the isolated mucosa). In the proximal gut, levels decrease from about $100 \mathrm{mg} / 100 \mathrm{~g}$ wet weight at birth to about $50 \mathrm{mg}$ in 12-day-old rats in the proximal gut, i.e. the region where we found the highest activities of gluconeogenic enzymes and maximal glucose production. In $20-$ day-old and older rats even lower glycogen content was found. 


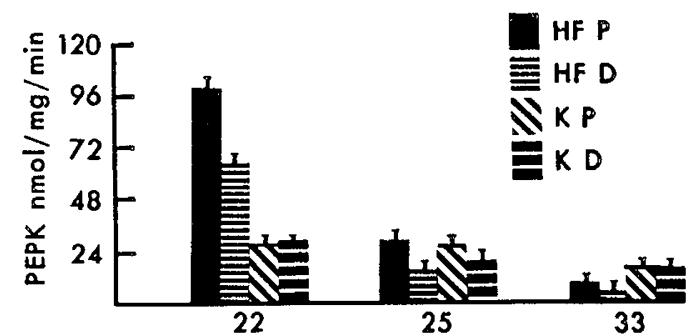

Fig. 6. Effect of weaning infant rats to a high fat or high carbohydrate diet on the 18 th postnatal day on phosphoenolpyruvate carboxykinase activity in the proximal and distal halves of the small intestinal mucosa on the 22nd, 25th, and 33rd days. From left to right for each age: high fat diet, proximal gut $(H F P)$; high fat diet, distal gut ( $H F D$ ); high carbohydrate diet, proximal gut $(K P)$; high carbohydrate diet, distal gut $(K D)$.

Again, this may be due to the fact that the whole gut and not the mucosa was assayed. In our experiments, glycogen levels decreased to about half the initial value during incubation; this is one of the reasons why we preferred to examine glucose formation by determining labeled glucose produced. We also found no appreciable label in glycogen at the end of incubation.

A relatively low rate of developing gut gluconeogenesis in comparison to the liver was found (see Reference 8). This is surprising considering the high levels of gluconeogenic enzyme activities. It suggests that the in vitro conditions employed for study are not optimal. This is supported by the fact that the rate of glucose formation decreases with the time of incubation; the rate is highest during the first $15 \mathrm{~min}$ and then decreases. Our preliminary data indicate that under certain conditions we may be able to improve the gluconeogenic capability of the mucosa. The absence of label in glycogen is not surprising since glycogen content is very low at the start and the amount of glucose incorporated may have been released again from the glycogen molecule.

Our data indicate that glucose formation from lactate can occur in suckling rat small intestinal mucosa but not in the mucosa from weaned animals. This is also true for the rabbit. This finding explains to some extent why the activities of the three key gluconeogenic enzymes are so high in the mucosa of the small intestine of infant rats and rabbits. However, this cannot be the whole explanation, since in the liver of the same animals the rate of glucose production is about 10 times higher. One can only speculate what other roles should be ascribed to the mucosal gluconeogenic enzymes in infancy. It is, of course, also possible that the newly formed glucose is utilized at such a high rate that the net increase in this substance is small.

Recently, it was reported that glycolysis, glucose oxidation, and lactate formation occur at slower rates in the mucosa of infant rats than in that of weaned animals (8). This would suggest that glucose utilization is low in infant rats, and this is generally true. However, the data reported by Kimura et al. (10) were obtained for the whole intestinal wall and not the isolated mucosa, and it is more than likely that the muscular layer of the gut metabolizes glucose differently than the mucosa. This is borne out by the absence of PEPcK in the muscular layer and the much greater contents of cyclic AMP in this layer in comparison to the mucosa (9).

One may pose the teleological question: why should the mucosa make glucose? We suggest that perhaps the new glucose serves as a substrate for the muscular layer, which may depend more on newly made substrate than later in life, since the external carbohydrate supply is limited in the suckling period. The paradox that the gut utilizes less glucose in infancy may only be apparent, since the ratio of mucosa to gut wall is much greater in infancy than after weaning. Hence data obtained on the whole gut in suckling rats reflect the preponderance of the mucosa to a greater extent than later in life.

The stimulatory effect of cyclic AMP is not surprising since it is an inducer of PEPcK (11) and an inhibitor of pyruvate kinase (12). It is probably the latter effect that leads to increased glucose formation. Cyclic AMP also causes glycogen breakdown, but it is unlikely that this mechanism is the cause of increased formation of labeled glucose (see above). The effect of TDGA is difficult to explain. It inhibits fatty acid oxidation (5), and hence one would expect gluconeogenesis to be suppressed. Since the gut obtains most of its energy from glutamate (13), it is possible that TDGA acts in this area by an as yet unexplained mechanism.

\section{REFERENCES}

1. Hahn P, Smale F-A 1984 Phosphoenolpyruvate carboxykinase in the small intestine of developing rodents. J Nutr 112:986-989

2. Westbury K. Hahn P 1984 Fructose-1,6-biphosphatase activity in the intestinal mucosa of developing rats. Am J Physiol 246:G683-G686

3. Menard D, Malo C 1978 Glucose-6-phosphatase activity in mouse small intestine during postnatal development Dev Biol 65:508-514

4. Carrol NV, Longley RW, Roe JH 1956 The determination of glycogen in liver and muscle by use of anthrone reagent. J Biol Chem 220:583-593

5. Tutwiler GF, Brentzel HJ 1982 Relation of oxidation of long-chain fatty acids to gluconeogenesis in the perfused liver of the guinea pig: effect of 2 tetradecylglycidic acid (McN-3802). Eur J Biochem 124:465-470

6. Deleted in proof.

7. Nathan P 1967 Glycogen content in the intestine of the fetal and neonatal rat Physiologist 10:260

8. Warnette-Hammond M, Lardy HR 1985 Catecholamine and vasopressin stimulation of gluconeogenesis from dehydroacetone in the presence of atractyloside. J Biol Chem 260:12647-12652

9. Hahn P, Cannon de Rodriguez W, Skala JP 1985 Effect of age and diet on cyclic nucleotide concentrations in the intestinal mucosa of developing rats. J Nutr (in press)

10. Kimura RE, Thulin G, Warshaw JB 1985 The effect of ketone bodies and fatty acid on intestinal glucose metabolism during development. Pediatr Res 18:575-578

11. Beale EG, Hartley JL, Granner DK $1982 \mathrm{~N}^{6}, \mathrm{O}^{2}$-dibutyryl cyclic AMP and glucose regulate the amount of messenger RNA coding for hepatic phosphoenolpyruvate carboxykinase (GTP). J Biol Chem 257:2022-2028

12. Munnich A, Marie J, Reach G, Vaulont S, Simon M-P, Kahn A 1984 In vivo hormonal control of L-type pyruvate kinase gene expression. Effects of glucagon, cyclic AMP, insulin, cortisone, and thyroid hormones on the dietary induction of mRNAs in the liver. J Biol Chem 259:10228-10231

13. Windmueller HG, Spaeth AE 1980 Respiratory fuels and nitrogen metabolism in vivo in small intestine of fed rats. Quantitative importance of glutamine, glutamate, and aspartate. J Biol Chem 255:107-112 